\title{
Treinamento de força para idosos: uma revisão integrativa
}

Nos últimos anos foi possível observar que a perspectiva de vida aumentou na população de idosos no Brasil e no mundo. Durante o processo de envelhecimento é comum que esse segmento etário apresentem os seguintes problemas de saúde: perda de densidade óssea (osteoporose) e massa muscular (sarcopenia), assim como articulações menos fortes e mais vulneráveis a danos, pois um tipo de acidente que acomete com frequência esse público são as quedas, que têm grande predominância entre as variáveis de ferimentos não intencionais. O objetivo desse estudo foi investigar por meio de revisão integrativa os efeitos do treinamento de força para a saúde de idosos. A busca por artigos se deu através das plataformas eletrônicas BVS e Scielo com os descritores elderly, strength traing, resistance training e os operadores AND e OR. Os artigos incluídos foram estudos publicados nos últimos 5 (cinco) anos, avaliados por meio de análise qualitativa que compreendeu relacionar os diferentes artigos selecionados com as questões da pesquisa a fim de atender seus objetivos. Dos artigos encontrados após análise de título e resumo foi totalizado 551 artigos. Dos selecionados foram excluídos da pesquisa 541 artigos. Após a leitura e avaliação restaram 10 artigos para construção desta revisão. Os resultados da pesquisa mostram que o treinamento de força pode ser eficaz para suprimir o processo de envelhecimento e possibilitar maior funcionalidade no dia-a-dia através do aumento da massa muscular, redução do percentual de gordura corporal, aumentando a força do indivíduo, de sua capacidade funcional, e melhora no estado cognitivo. Diante do estudo conclui-se que o treinamento de força pode ser muito eficaz para a manutenção da saúde e uma melhor qualidade de vida dos idosos, pois possibilita maior funcionalidade para atividades diárias e benefícios para saúde física e mental, entretanto devese respeitar as variáveis do treinamento para não gerar complicações, pois é necessário novos estudos mais aprofundados que possam comprovar os benefícios do treinamento de força para a saúde de idosos.

Palavras-chave: Envelhecimento; Treinamento de Força; Qualidade de Vida.

\section{Strength training for the elderly: an integrative review}

\begin{abstract}
In the last few years it was possible to observe that the perspective of life has increased in the elderly population in Brazil and in the world. During the aging process it is common for this age group to have the following health problems: loss of bone density (osteoporosis) and muscle mass (sarcopenia), as well as less strong joints and more vulnerable to damage, as a type of accident that affects this audience is often falls, which are highly prevalent among variables of unintentional injuries. The aim of this study was to investigate, through an integrative review, the effects of strength training on the health of the elderly. The search for articles took place through the electronic platforms VHL and SciELO with the descriptors elderly, strength traing, resistance training and the operators AND and OR. The articles included were studies published in the last 5 (five) years, assessed through qualitative analysis that included relating the different articles selected to the research questions in order to meet their objectives. A total of 551 articles were found after analyzing the title and summary. From the selected, 541 articles were excluded from the research. After reading and evaluating, 10 articles remained for the construction of this review. The results of the research show that strength training can be effective to suppress the aging process and enable greater functionality in daily life by increasing muscle mass, reducing the percentage of body fat, increasing the strength of the individual, their functional capacity, and improvement in cognitive status. In view of the study, it is concluded that strength training can be very effective for maintaining health and a better quality of life for the elderly, as it allows greater functionality for daily activities and benefits for physical and mental health, however, one must respect the training variables so as not to generate complications, as further in-depth studies are needed to prove the benefits of strength training for the health of the elderly.
\end{abstract}

Keywords: Aging; Strength Training; Quality of life.

Topic: Educação Física

Reviewed anonymously in the process of blind peer.
Received: 09/02/2020

Approved: $20 / 05 / 2020$
Cícero Cleber Brito Pereira (iD

Centro Universitário Vale do Salgado, Brasil http://lattes.cnpq.br/6554764959422181

http://orcid.org/0000-0002-9402-9810

cicerocleber@univs.edu.br

Poliana Granjeiro Brasil (iD

Centro Universitário Vale do Salgado, Brasil http://lattes.cnpq.br/3038480219737326

http://orcid.org/0000-0002-5613-5073

polianabrasil.fvs@hotmail.com

Gabriela Gomes de Oliveira Bezerra (iD)

Centro Universitário Dr. Leão Sampaio, Brasil

http://lattes.cnpq.br/6330341118187369

http://orcid.org/0000-0002-9724-708X

gabriela.o.bezerra@gmail.com
Marcos Antônio Araújo Bezerra (iD)

Centro Universitário Dr. Leão Sampaio, Brasil

http://lattes.cnpq.br/4643352879633283

http://orcid.org/0000-0002-3385-4024

marcosantonio@leaosampaio.edu.br
Referencing this:

PEREIRA, C. C. B.; BRASIL, P. G.; BEZERRA, G. G. O.; BEZERRA, M. A. A Treinamento de força para idosos: uma revisão integrativa. Medicus, v.2, n.2, p.6-17, 2020. DOI: http://doi.org/10.6008/CBPC26746484.2020 .002 .0002 


\section{INTRODUÇÃO}

O treinamento de força, também chamado como treinamento contra resistência ou treinamento com pesos, fez-se uma das formas mais comuns de exercícios para aperfeiçoar a aptidão física de um sujeito e para o condicionamento físico de atletas (FLECK et al., 2017). A musculação é uma prática amparada nos princípios de treinamento com pesos e é um meio eficiente no estímulo de respostas fisiológicas ao exercício. É uma atividade física desenvolvida por meio de exercícios analíticos, que usam a resistência progressiva oferecida por recursos materiais, como barras, anilhas, halteres, aglomerados, módulos, extensores, peças lastradas, ou até mesmo o próprio corpo (PINHEIRO et al., 2018).

O envelhecimento é um processo complexo e inclui vários fatores. A variabilidade de cada indivíduo (genética e ambiental) acaba evitando o estabelecimento de padrões. Por isso, o uso apenas do tempo da idade cronológica como medida encobre um amplo conjunto de aspectos. A idade em si não define o envelhecimento, ela é apenas uma das variáveis presentes no processo de desenvolvimento, servindo como um parâmetro da passagem do tempo (PINTO et al., 2017).

O processo de envelhecer é considerado como uma preocupação do indivíduo em relação ao passar do tempo. Assim a sociedade acaba desvalorizando o envelhecimento, não aceitando como um processo inevitável até a morte. A terceira idade causa sentimentos negativos nas pessoas devido as consequências provocadas por esses fenômenos, que ocorrem ao longo da vida (COLUSSI et al., 2019).

Durante o processo de envelhecimento, mesmo na falta de doença crônica, está relacionado a uma variedade de alterações biológicas que podem colaborar para reduções na massa muscular esquelética, força e função, provocando uma diminuição da resistência fisiológica e fragilidades a eventos desastrosos. Por volta dos 60 anos de idade é vista uma diminuição de força máxima muscular entre 30 e 40\%, o que equivale a uma perda de força de aproximadamente $6 \%$ por década dos 35 aos 50 anos de idade, daí então, 10\% por década (ROCHA et al., 2019).

Entretanto neste período é comum que os idosos apresentem problemas de saúde como perda da densidade óssea e massa muscular, tornando as articulações menos fortes e mais vulneráveis a danos. E com isso pode-se apresentar: problemas em realizar atividades físicas, antes consideradas fáceis, como subir escada, substituir uma lâmpada e transportar compras ou malas, quedas frequentes, normalmente, quando a perda muscular já está em estado adiantado, perda de equilíbrio ao andar em terrenos irregulares, como ruas com buracos (BARBOSA et al., 2019). De acordo com Cyrino et al. (2019) o treinamento de força pode gerar um aumento na massa muscular e densidade óssea. Diante do exposto tem-se como problema de pesquisa: Quais os efeitos do treinamento de força sobre a saúde de idosos?.

Um tipo de acidente que acomete com frequência idosos que apresentam perda de densidade óssea (osteoporose) e massa muscular (sarcopenia) são as quedas. As quedas têm grande predominância entre as variáveis de ferimentos não intencionais. São classificadas como (CID-9) na Classificação Internacional das doenças (WHO, 2008).

As quedas são uma das principais razões de internações no Brasil, principalmente entre a população 
idosa, sendo assim, um problema de saúde pública em crescimento. Em 2013 houveram 93.312 internações por quedas em indivíduos com mais de 60 anos, registradas pelo Sistema Único de Saúde (SUS). Relativamente à mortalidade, dados mostram que no mesmo ano 8.775 morreram por este motivo no país (ABREU et al., 2018).

Este estudo se faz importante para a comunidade acadêmica visto que envelhecimento é um processo irreversível, mas é possível envelhecer com saúde física e mental. Nesta perspectiva, torna-se fundamental o desenvolvimento de estudos que possam trazer melhorias na estrutura de vida desta população, promovendo medidas que possam vir de recomendações tanto de políticas públicas, quanto de procedimentos sociais que beneficiem pessoas idosas proporcionando melhoras na qualidade de vida. Assim, procurar na literatura a importância do treinamento de força na terceira idade é necessário para facilitar contribuições para essa população (CONFORTIN et al., 2017).

Para a sociedade esta pesquisa é relevante porque o número de pessoas idosas vem aumentando de modo gradativo no Brasil. A população brasileira manteve a propensão de envelhecimento dos últimos anos e adquiriu 4,8 milhões de idosos desde 2012, ultrapassando o número de 30,2 milhões em 2017. Em 2012, no Brasil a população com 60 anos ou mais era de 25,4 milhões. Os 4,8 milhões de novos idosos em cinco anos representam um aumento de $18 \%$ dessa faixa etária, que tem sido cada vez mais significante no Brasil. As mulheres são maior parte considerável nesse grupo, com 16,9 milhões (56\% dos idosos), em conta partida os homens idosos são 13,3 milhões (44\% do grupo) (IBGE, 2018).

A prática do treinamento de força para indivíduos idosos consiste em um importante método para a melhoria da atividade física diária, da independência e, portanto, da qualidade de vida desses sujeitos. Melhoras na força e na potência muscular, importante para a manutenção da independência e para diminuição das quedas de idosos, podem ser vistos após poucas semanas de treinamento (SILVA et al., 2019). O objetivo desse estudo foi investigar, por meio de revisão integrativa, os efeitos do treinamento de força para a saúde de idosos.

\section{METODOLOGIA}

\section{Caracterização do estudo}

Esse estudo trata-se de uma revisão integrativa com abordagem qualitativa, que tem por objetivo, unir o conhecimento científico, antes construído sobre o tema proposto na pesquisa. Assim, busca avaliar os índices disponíveis a colaboração para o desenvolvimento da temática (ESTRELA, 2018).

\section{Instrumentos e Procedimentos}

A pesquisa de artigos científicos foi realizada por meio de busca eletrônica nas seguintes bases de dados: BVS e SciELO. Os descritores (DeCS) utilizados para a busca de estudos nas bases de dados referidas foram: 'elderly', 'strength training', 'resistance training' e os operadores AND e OR. Em virtude das características particulares de cada base de dados, as estratégias de busca são adaptadas conforme o 
objetivo e os critérios de inclusão e exclusão deste estudo.

Os critérios de inclusão utilizados na seleção dos artigos foram os seguintes: Estudos publicados nos últimos 5 (cinco) anos; Estudos que abordam os efeitos do treinamento de força para indivíduos idosos; Estudos publicados em revistas com qualis $\geq$ B2; Já os critérios de exclusão foram: Estudos duplamente indexados nas bases de dados; Acesso integral aos estudos não é gratuito; Estudos com amostra $<40$ indivíduos; Estudos não realizados com humanos.

\section{Análise de Dados}

Os artigos incluídos neste estudo foram avaliados por meio de análise qualitativa. Segundo Lakatos et al. (2006), a análise qualitativa se caracteriza por ser a abordagem que estabelece relações entre características que podem ser observadas e experimentalmente verificáveis de um objeto de estudo. A fase de análise dos artigos compreendeu em relacionar os diferentes artigos selecionados com as questões de pesquisa, a fim de conseguir contribuições para responder a mesma e assim atingir o objetivo do estudo. Isso permitirá visualizar de forma clara a questão da pesquisa respondida pelos artigos selecionados. Dessa maneira, buscou-se responder à pergunta de pesquisa tendo como base os artigos que se relacionam a ela.

\section{RESULTADOS E DISCUSSÃO}

Dos 10 (dez) artigos incluídos nesta revisão integrativa, a predominância do tempo de duração das intervenções foi de 12 semanas. Em relação a amostra, em 62,5\% dos estudos a mesma era composta apenas por indivíduos do sexo feminino, em $25 \%$ indivíduos de ambos os sexos (feminino e masculino) e em $12,5 \%$ indivíduos somente do sexo masculino.

Embora as intervenções realizadas nos estudos diferenciem quanto as variáveis do treinamento de força (método de treinamento, número de séries, intensidade dos exercícios, tempo de intervalo de recuperação entre as séries e exercícios, ordem dos exercícios ou velocidade de execução do movimento), em todas foi possível observar efeitos positivos do treinamento de força para idosos, todavia, alguns autores além de citar os benefícios citaram também os malefícios que o mesmo tem a capacidade de provocar.

Foi constado através dos estudos de Santos et al. (2019), Cardalda et al. (2019) efeitos positivos do treinamento de força em parâmetros diversos como: força muscular, potência muscular, resistência muscular, massa muscular, qualidade de vida, estado cognitivo, independência funcional, estabilidade, composição corporal e aptidão física.

Segundo os estudos de Cardalda et al. (2019) e Santos et al. (2019) o treinamento de força a partir de 12 semanas de intervenção pode gerar alterações em fatores associados ao processo de envelhecimento (degeneração do estado cognitivo e degeneração da independência funcional), e estas modificações podem prevenir ou atrasar o declínio no estado cognitivo.

De acordo com o estudo de Cardalda et al. (2019) o treinamento de força vem obtendo progressivamente uma função valiosa na prevenção e tratamento de indivíduos com declínio cognitivo. No estudo, os indivíduos que realizaram treinamento de força obtiveram melhora de $85,71 \%$ na função social, 
$16,67 \%$ no estado emocional, $53,10 \%$ no bem-estar emocional.

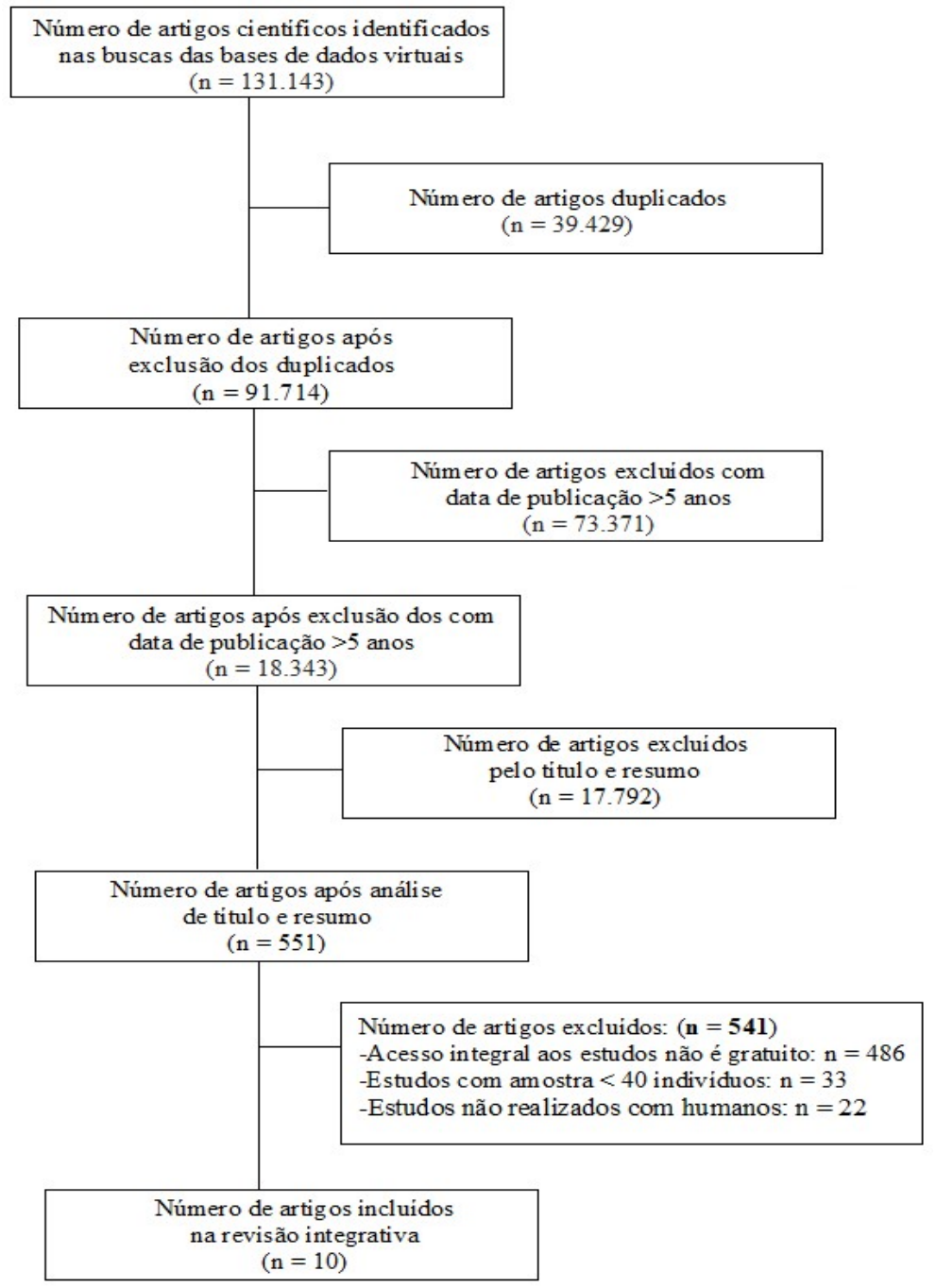

Figura 1: Processo de seleção de artigos nas bases de dados BVS e SciELO. Fonte: Baseado em Domholdt, (2005); Law et al. (2002); e Magee (1998).

Tabela 1: Descrição dos artigos selecionados.

\begin{tabular}{|c|c|c|c|c|c|c|}
\hline $\begin{array}{c}\text { AUTOR E } \\
\text { ANO } \\
\text { DE } \\
\text { PUBLICAÇÃO } \\
\end{array}$ & OBJETIVO & $\begin{array}{c}\text { AMOSTRA E } \\
\text { PRINCIPAIS } \\
\text { FERRAMENTAS } \\
\text { DO ESTUDO }\end{array}$ & $\begin{array}{l}\text { QUALIS DA } \\
\text { REVISTA }\end{array}$ & INTERVENÇÃO & RESULTADOS & CONCLUSÃO \\
\hline $\begin{array}{l}\text { ARAGÃO- } \\
\text { SANTOS et } \\
\text { al., } 2019\end{array}$ & $\begin{array}{l}\text { Comparar os efeitos } \\
\text { do treinamento } \\
\text { funcional e } \\
\text { tradicional de força } \\
\text { sobre a força } \\
\text { dinâmica máxima, } \\
\text { potência muscular, } \\
\text { resistência muscular e } \\
\text { força isométrica em } \\
\text { idosos. }\end{array}$ & $\begin{array}{l}44 \text { idosas } \\
\text { divididas } \\
\text { aleatoriamente } \\
\text { em três grupos: } \\
\text { treinamento } \\
\text { funcional (TF; N. = } \\
\text { 18), treinamento } \\
\text { tradicional (TT; N. } \\
=15 \text { ) e grupo } \\
\text { controle (GC; N. = } \\
\text { 11). 1RM; } \\
\text { Speedometer; } \\
\text { HD; DC; } 30-s \text { Chair } \\
\text { Stand; Arm Curl } \\
\text { Test }\end{array}$ & $\begin{array}{l}\text { The Journal } \\
\text { of Sports } \\
\text { Medicine } \\
\text { and Physical } \\
\text { Fitness: A2 }\end{array}$ & $\begin{array}{l}\text { Protocolos de } \\
\text { treino com } \\
\text { duração de } 12 \\
\text { semanas. A } \\
\text { frequência foi de } \\
\text { três sessões } \\
\text { semanais } \\
\text { realizadas em dias } \\
\text { não consecutivos } \\
\text { com uma duração } \\
\text { de } \\
\text { aproximadament } \\
\text { e } 50 \text { minutos. }\end{array}$ & $\begin{array}{l}\text { Ambos os grupos } \\
\text { experimentais } \\
\text { melhoraram } \\
\text { significativament } \\
\text { e em força, } \\
\text { potência, força } \\
\text { isométrica e } \\
\text { resistência. }\end{array}$ & $\begin{array}{l}\text { Ambos os } \\
\text { protocolos de } \\
\text { treinamento foram } \\
\text { igualmente eficazes } \\
\text { para melhorar as } \\
\text { diferentes } \\
\text { manifestações de } \\
\text { força em idosos. }\end{array}$ \\
\hline $\begin{array}{l}\text { CARDALDA } \\
\text { et al., } 2019\end{array}$ & $\begin{array}{l}\text { Verificar os diferentes } \\
\text { efeitos de dois } \\
\text { programas de } \\
\text { exercício físico } \\
\text { (treinamento de } \\
\text { força-TF e } \\
\text { treinamento } \\
\text { calistênico-TC) sobre }\end{array}$ & $\begin{array}{l}77 \text { idosos } \\
\text { institucionalizado } \\
\text { s e fragilizados } \\
\text { divididos em três } \\
\text { grupos: (TF = 25), } \\
\text { (TC = 23) e grupo } \\
\text { controle (GC = 29) } \\
\text { IMC; ES; }\end{array}$ & $\begin{array}{l}\text { Science } \\
\text { Direct: A1 }\end{array}$ & $\begin{array}{l}\text { O grupo TF } \\
\text { realizou um } \\
\text { programa de } \\
\text { força com faixas } \\
\text { elásticas durante } \\
12 \text { semanas; O TC } \\
\text { realizou um } \\
\text { programa de }\end{array}$ & $\begin{array}{l}\text { O programa TF } \\
\text { gerou alguma } \\
\text { melhora no } \\
\text { estado cognitivo } \\
\text { e na } \\
\text { independência } \\
\text { funcional, } \\
\text { enquanto no }\end{array}$ & $\begin{array}{l}\text { O exercício físico, } \\
\text { seja o programa de } \\
\text { força ou o } \\
\text { programa } \\
\text { multicalistênico, é } \\
\text { um método eficaz } \\
\text { para melhorar e } \\
\text { manter a saúde, o }\end{array}$ \\
\hline
\end{tabular}


PEREIRA, C. C. B.; BRASIL, P. G.; BEZERRA, G. G. O.; BEZERRA, M. A. A.

\begin{tabular}{|c|c|c|c|c|c|c|}
\hline & $\begin{array}{l}\text { o estado cognitivo, } \\
\text { funcionalidade, } \\
\text { estabilidade e saúde } \\
\text { geral de idosos } \\
\text { institucionalizados } \\
\text { em idade frágil }\end{array}$ & $\begin{array}{l}\text { Stadiometer; PP; } \\
\text { Barthel Index; } \\
\text { FTSTS; SF-12; } \\
\text { MMSET; Pfeiffer } \\
\text { Test; }\end{array}$ & & $\begin{array}{l}\text { exercícios } \\
\text { multicalistenicos } \\
\text { e o GC não } \\
\text { realizou nenhum } \\
\text { exercício físico. }\end{array}$ & $\begin{array}{l}\text { componente } \\
\text { físico e mental do } \\
\text { S-12, uma } \\
\text { melhora } \\
\text { significativa foi } \\
\text { gerada. O } \\
\text { programa TC } \\
\text { demonstrou uma } \\
\text { tendência à } \\
\text { estabilização dos } \\
\text { parâmetros } \\
\text { mencionados } \\
\text { acima, enquanto } \\
\text { o GC demonstrou } \\
\text { uma tendência à } \\
\text { deterioração }\end{array}$ & $\begin{array}{l}\text { estado cognitivo, a } \\
\text { independência } \\
\text { funcional e a } \\
\text { estabilidade em } \\
\text { pessoas } \\
\text { institucionalizadas } \\
\text { em idade frágil. }\end{array}$ \\
\hline $\begin{array}{l}\text { MAZINI } \\
\text { FILHO et al., } \\
2018\end{array}$ & $\begin{array}{l}\text { Investigar os efeitos } \\
\text { do treinamento de } \\
\text { força em circuito } \\
\text { sobre a força } \\
\text { muscular, autonomia } \\
\text { funcional e } \\
\text { indicadores } \\
\text { antropométricos de } \\
\text { idosos }\end{array}$ & $\begin{array}{l}65 \text { mulheres } \\
\text { divididas em dois } \\
\text { grupos: } \\
\text { treinamento de } \\
\text { força (GT, N. = 34) } \\
\text { e grupo controle } \\
\text { (GC, N. = 31) } \\
\text { Mechanical Scale; } \\
\text { Stadiometer; } \\
\text { WHR; RJP; 10RM; }\end{array}$ & $\begin{array}{l}\text { The Journal } \\
\text { of Sports } \\
\text { Medicine } \\
\text { and Physical } \\
\text { Fitness: A2 }\end{array}$ & $\begin{array}{l}\text { O grupo de } \\
\text { treinamento de } \\
\text { força foi } \\
\text { submetido a um } \\
\text { programa de } \\
\text { treinamento em } \\
\text { circuito, três dias } \\
\text { por semana, por } \\
\text { um período de } 12 \\
\text { semanas. Em } \\
\text { cada sessão de } \\
\text { treinamento, o } \\
\text { circuito foi } \\
\text { repetido três } \\
\text { vezes. Em cada } \\
\text { circuito, todos os } \\
\text { exercícios foram } \\
\text { realizados uma } \\
\text { vez, com } 8 \text { a } 12 \\
\text { repetições por } \\
\text { exercício, com } \\
\text { intervalos de } 30 \\
\text { segundos entre } \\
\text { cada exercício. }\end{array}$ & $\begin{array}{l}\text { O GT mostrou } \\
\text { alterações } \\
\text { significativas na } \\
\text { composição } \\
\text { corporal após } 12 \\
\text { semanas, como } \\
\text { diminuições no } \\
\text { peso corporal e } \\
\text { diminuições no } \\
\text { abdominal, } \\
\text { circunferência da } \\
\text { cintura, quadril e } \\
\text { da razão cintura- } \\
\text { quadril. Para } \\
\text { autonomia } \\
\text { funcional, o GT } \\
\text { mostrou } \\
\text { aumentos após } \\
\text { 12 semanas no } \\
\text { suporte de } \\
\text { cadeira de } 30 \\
\text { segundos, } \\
\text { caminhada de } \\
\text { seis minutos. O } \\
\text { GT também } \\
\text { mostrou } \\
\text { aumentos na } \\
\text { força muscular. } \\
\text { Para o GC, a } \\
\text { composição } \\
\text { corporal, a } \\
\text { autonomia } \\
\text { funcional e a } \\
\text { força muscular } \\
\text { não melhoraram } \\
\text { em nenhum } \\
\text { momento } \\
\end{array}$ & $\begin{array}{l}\text { O treinamento de } \\
\text { força em circuito } \\
\text { fornece melhorias } \\
\text { significativas na } \\
\text { força muscular, } \\
\text { desempenho } \\
\text { funcional e } \\
\text { indicadores } \\
\text { antropométricos } \\
\text { em mulheres idosas } \\
\text { sedentárias. }\end{array}$ \\
\hline $\begin{array}{l}\text { SMOLAREK } \\
\text { et al., } 2016\end{array}$ & $\begin{array}{l}\text { Verificar o efeito de } \\
\text { um programa de } \\
\text { exercícios resistidos } \\
\text { de } 12 \text { semanas na } \\
\text { aptidão física geral e } \\
\text { nas capacidades } \\
\text { cognitivas de } \\
\text { mulheres idosas e } \\
\text { sedentárias }\end{array}$ & $\begin{array}{l}\text { Vinte e nove } \\
\text { mulheres } \\
\text { divididas em dois } \\
\text { grupos. O grupo } \\
\text { controle foi } \\
\text { composto por } 8 \\
\text { idosas e o grupo } \\
\text { de treinamento } \\
\text { de força foi } \\
\text { composto por } 21 \\
\text { idosas submetidas } \\
\text { a um programa de } \\
\text { exercícios } \\
\text { resistidos Digital } \\
\text { Scale; } \\
\text { Stadiometer; } \\
\text { 'SRT'; 'MNAC' } \\
\text { Test; Chair Test; } \\
\text { MoCA; }\end{array}$ & $\begin{array}{l}\text { Clinical } \\
\text { Intervention } \\
\text { s In Aging: } \\
\text { A1 }\end{array}$ & $\begin{array}{l}\text { Programa de } \\
\text { exercícios } \\
\text { resistidos com } \\
\text { duração de } 12 \\
\text { semanas definido } \\
\text { por } 12 \text { exercícios } \\
\text { de membros } \\
\text { superiores e } \\
\text { inferiores } \\
\text { combinados em } 3 \\
\times 10 \text { repetições } \\
\text { com intervalo de } \\
1 \text { minuto entre as } \\
\text { repetições e dois } \\
\text { minutos de } \\
\text { descanso entre os } \\
\text { exercícios (três } \\
\text { vezes/semana) }\end{array}$ & $\begin{array}{l}\text { O grupo de idosas } \\
\text { mostrou } \\
\text { aumentos } \\
\text { significativos na } \\
\text { força média da } \\
\text { parte superior do } \\
\text { corpo }(58 \%) \text {, na } \\
\text { força inferior do } \\
\text { corpo (68\%) e na } \\
\text { capacidade } \\
\text { cognitiva (19\%). }\end{array}$ & $\begin{array}{l}\text { Exercícios regulares } \\
\text { de resistência } \\
\text { proporcionam } \\
\text { ganhos } \\
\text { significativos na } \\
\text { força do corpo } \\
\text { superior e inferior, } \\
\text { concomitantement } \\
\text { e a melhorias } \\
\text { positivas nas } \\
\text { capacidades } \\
\text { cognitivas de } \\
\text { mulheres idosas, } \\
\text { trazendo maior } \\
\text { qualidade de vida. }\end{array}$ \\
\hline $\begin{array}{l}\text { LOPEZ et al., } \\
2017\end{array}$ & $\begin{array}{l}\text { Avaliar } \\
\text { sistematicamente o } \\
\text { efeito do treinamento } \\
\text { resistido (TR) sozinho }\end{array}$ & $X$ & $\begin{array}{l}\text { Clinical } \\
\text { Intervention } \\
\text { s in Aging: } \\
\text { A1 }\end{array}$ & $X$ & $\begin{array}{l}\text { TR isolado ou em } \\
\text { treinamento } \\
\text { multimodal pode } \\
\text { induzir aumentos } \\
\end{array}$ & $\begin{array}{l}\text { TR supervisionado e } \\
\text { controlado } \\
\text { represente uma } \\
\text { intervenção eficaz }\end{array}$ \\
\hline
\end{tabular}




\begin{tabular}{|c|c|c|c|c|c|c|}
\hline & $\begin{array}{l}\text { ou combinado com a } \\
\text { intervenção } \\
\text { multimodal sobre a } \\
\text { hipertrofia muscular, } \\
\text { força máxima, } \\
\text { potência, } \\
\text { desempenho } \\
\text { funcional e incidência } \\
\text { de quedas em idosos } \\
\text { fisicamente frágeis. }\end{array}$ & & & & $\begin{array}{l}\text { de } 6,6 \% \text { na força } \\
\text { máxima; } 3,4 \% \text { em } \\
\text { massa muscular, } \\
8,2 \% \text { em potência } \\
\text { muscular, } 4,7 \% \\
\text { em capacidade } \\
\text { funcional e risco } \\
\text { de quedas. }\end{array}$ & $\begin{array}{l}\text { no tratamento da } \\
\text { fragilidade. }\end{array}$ \\
\hline $\begin{array}{l}\text { CAVALCANTE } \\
\text { et al., } 2018\end{array}$ & $\begin{array}{l}\text { Comparar o efeito de } \\
\text { diferentes programas } \\
\text { de treinamento de } \\
\text { resistência (TR) sobre } \\
\text { o total de gordura } \\
\text { androide, ginoide e } \\
\text { de tronco em idosas } \\
\text { com } \\
\text { sobrepeso/obesidade } \\
\text {. }\end{array}$ & $\begin{array}{l}57 \text { mulheres } \\
\text { idosas com } \\
\text { sobrepeso/obesas } \\
\text { foram } \\
\text { aleatoriamente } \\
\text { designadas para } \\
\text { um dos três } \\
\text { grupos: um grupo } \\
\text { realizando TR } \\
\text { duas vezes por } \\
\text { semana (G2X), um } \\
\text { grupo realizando } \\
\text { TR três vezes por } \\
\text { semana (G3X), e } \\
\text { um grupo de } \\
\text { controle sem } \\
\text { exercício (GC) } \\
\text { DXA; }\end{array}$ & $\begin{array}{l}\text { International } \\
\text { Journal of } \\
\text { Sports } \\
\text { Medicine: A1 }\end{array}$ & $\begin{array}{l}\text { Ambos os grupos } \\
\text { de treinamento } \\
\text { realizaram o } \\
\text { mesmo programa } \\
\text { de TR de } 12 \\
\text { semanas, } \\
\text { composto por } 8 \\
\text { exercícios que } \\
\text { treinaram todos } \\
\text { os principais } \\
\text { grupos } \\
\text { musculares. }\end{array}$ & $\begin{array}{l}\text { Após o período } \\
\text { de intervenção, } \\
\text { G2X e G3X } \\
\text { demonstraram } \\
\text { reduções } \\
\text { significativas na } \\
\text { adiposidade em } \\
\text { comparação ao } \\
\text { GC para gordura } \\
\text { corporal total } \\
\text { (gordura } \\
\text { androide, ginoide } \\
\text { e de tronco), sem } \\
\text { diferenças } \\
\text { significativas } \\
\text { entre os grupos } \\
\text { de treinamento. }\end{array}$ & $\begin{array}{l}\text { Um programa de TR } \\
\text { de } 12 \text { semanas de } \\
\text { baixo volume, } \\
\text { realizado duas ou } \\
\text { três vezes por } \\
\text { semana, causa } \\
\text { reduções } \\
\text { significativas na } \\
\text { adiposidade } \\
\text { corporal. }\end{array}$ \\
\hline $\begin{array}{l}\text { STOEVER et } \\
\text { al., } 2018\end{array}$ & $\begin{array}{l}\text { Investigar a influência } \\
\text { do treinamento } \\
\text { resistido (TR) na } \\
\text { função física de } \\
\text { idosos obesos com } \\
\text { sarcopenia. }\end{array}$ & $\begin{array}{l}\text { Idosos } \\
\text { fisicamente } \\
\text { inativos e obesos, } \\
\text { sem doenças } \\
\text { graves. Eles foram } \\
\text { divididos em um } \\
\text { grupo com } \\
\text { sarcopenia (SAR, } \\
n=28 \text { ) e um } \\
\text { grupo sem ou } \\
\text { com pré- } \\
\text { sarcopenia (NSAR, } \\
n=20 \text { ) } \\
\text { SPPB; PPT; Hand } \\
\text { Dynamometer; } \\
\text { BA; SMI; FRT; }\end{array}$ & $\begin{array}{l}\text { International } \\
\text { Journal of } \\
\text { Sports } \\
\text { Medicine: A1 } \\
\text { Journal of } \\
\text { Geriatric } \\
\text { Physical } \\
\text { Therapy: A1 }\end{array}$ & $\begin{array}{l}\text { Durante o } \\
\text { período12 } \\
\text { semanas TR } \\
\text { progressivo } \\
\text { realizado duas } \\
\text { vezes por } \\
\text { semana, com } 60 \% \\
\text { a } 85 \% \text { da força } \\
\text { máxima com } 3 \\
\text { séries de } 8 \text { a } 12 \\
\text { repetições. }\end{array}$ & $\begin{array}{l}\text { Os participantes } \\
\text { do grupo SAR } \\
\text { conseguiram } \\
\text { aumentar } \\
\text { significativament } \\
\text { e o desempenho } \\
\text { em força de } \\
\text { preensão manual, } \\
\text { velocidade da } \\
\text { marcha, escore } \\
\text { SPPB e escore } \\
\text { PPT. Os } \\
\text { participantes do } \\
\text { grupo NSAR } \\
\text { também foram } \\
\text { capazes de } \\
\text { melhorar seu } \\
\text { desempenho já } \\
\text { bom em força de } \\
\text { preensão manual, } \\
\text { velocidade da } \\
\text { marcha, escore } \\
\text { SPPB e escore } \\
\text { PPT. No entanto, } \\
\text { os participantes } \\
\text { de ambos os } \\
\text { grupos não } \\
\text { puderam } \\
\text { aumentar os } \\
\text { resultados do } \\
\text { Índice De Massa } \\
\text { Muscular } \\
\text { Esquelética e do } \\
\text { Teste de Alcance } \\
\text { Funcional. } \\
\end{array}$ & $\begin{array}{l}\text { Os participantes de } \\
\text { ambos os grupos } \\
\text { melhoraram seu } \\
\text { desempenho físico } \\
\text { em vários } \\
\text { parâmetros após o } \\
\text { treinamento. Os } \\
\text { resultados também } \\
\text { mostraram que } \\
\text { adultos obesos com } \\
\text { sarcopenia se } \\
\text { beneficiaram do } \\
\text { treinamento de } \\
\text { força. }\end{array}$ \\
\hline $\begin{array}{l}\text { PINHEIRO et } \\
\text { al., } 2018\end{array}$ & $\begin{array}{l}\text { Buscar } \\
\text { sistematicamente na } \\
\text { literatura evidências } \\
\text { de hipertrofia } \\
\text { muscular em pessoas } \\
\text { idosas por meio do } \\
\text { treinamento de } \\
\text { resistência. }\end{array}$ & $X$ & $\begin{array}{l}\text { Fisioterapia } \\
\text { Brasil: B2 }\end{array}$ & $x$ & $\begin{array}{l}\text { Os estudos } \\
\text { demonstram que } \\
\text { é possível } \\
\text { melhorar a massa } \\
\text { muscular em } \\
\text { treinamento de } \\
\text { resistência em } \\
\text { idosos, uma vez } \\
\text { que os exercícios } \\
\text { apresentem a } \\
\text { dose correta: } \\
\text { intensidade, } \\
\text { volume, carga } \\
\end{array}$ & $\begin{array}{l}\text { Sugere-se que para } \\
\text { hipertrofia } \\
\text { muscular em } \\
\text { idosos, os } \\
\text { protocolos de } \\
\text { treinamento } \\
\text { resistidos tenham } \\
\text { em média } 12 \\
\text { semanas de } \\
\text { treinamento, com } \\
\text { frequência de duas } \\
\text { a três vezes por } \\
\text { semana, que }\end{array}$ \\
\hline
\end{tabular}




\begin{tabular}{|c|c|c|c|c|c|c|}
\hline & & & & & $\begin{array}{l}\text { apropriada, } \\
\text { utilizando uma } \\
\text { investigação } \\
\text { sensível. }\end{array}$ & $\begin{array}{l}\text { apresentem cinco } \\
\text { exercícios } \\
\text { realizados em três } \\
\text { séries de oito a } 12 \\
\text { repetições e cargas } \\
\text { superiores a } 60 \% \\
\text { 1RM para que } \\
\text { ocorra a hipertrofia } \\
\text { muscular. }\end{array}$ \\
\hline $\begin{array}{l}\text { PINHEIRO et } \\
\text { al., } 2018 \\
\text { RIBEIRO et } \\
\text { al., } 2015\end{array}$ & $\begin{array}{l}\text { Analisar o efeito do } \\
\text { treinamento resistido } \\
\text { (TR) realizado em } \\
\text { diferentes } \\
\text { frequências semanais } \\
\text { sobre a flexibilidade } \\
\text { em mulheres mais } \\
\text { velhas }\end{array}$ & $\begin{array}{l}\text { Cinquenta e três } \\
\text { mulheres idosas } \\
\text { foram } \\
\text { aleatoriamente } \\
\text { designadas para } \\
\text { realizar TR, duas } \\
(\mathrm{n}=28 \text {; grupo } \\
\text { 'G2x') ou três ( } \mathrm{n}= \\
25 \text {; grupo 'G3x') } \\
\text { vezes por semana } \\
\text { CES; Kim } \\
\text { Equation; } \\
\text { Fleximeter; }\end{array}$ & $\begin{array}{l}\text { Clinical } \\
\text { Intervention } \\
\text { s in Aging: } \\
\text { A1 }\end{array}$ & $\begin{array}{l}\text { O programa de TR } \\
\text { compreendeu } \\
\text { oito exercícios } \\
\text { nos quais os } \\
\text { participantes } \\
\text { realizaram um } \\
\text { conjunto de } 10 \text { a } \\
15 \text { repetições no } \\
\text { máximo por um } \\
\text { período de } 12 \\
\text { semanas. }\end{array}$ & $\begin{array}{l}\text { Observou-se } \\
\text { interação } \\
\text { significativa grupo } \\
\text { a tempo para } \\
\text { flexão frontal do } \\
\text { quadril, na qual } \\
\text { G3x apresentou } \\
\text { um aumento } \\
\text { maior que o G2x. } \\
\text { Ambos os grupos } \\
\text { aumentaram a } \\
\text { flexibilidade na } \\
\text { extensão cervical, } \\
\text { flexão do quadril } \\
\text { direito e flexão do } \\
\text { quadril esquerdo. } \\
\text { Não foram } \\
\text { observadas } \\
\text { diferenças } \\
\text { estatisticamente } \\
\text { significativas para } \\
\text { o aumento da } \\
\text { massa muscular } \\
\text { esquelética entre } \\
\text { o treinamento } \\
\text { três e duas vezes } \\
\text { por semana. }\end{array}$ & $\begin{array}{l}\text { Doze semanas de } \\
\text { TR melhoram a } \\
\text { flexibilidade de } \\
\text { diferentes } \\
\text { movimentos } \\
\text { articulares em } \\
\text { mulheres mais } \\
\text { velhas, e a maior } \\
\text { frequência induz } \\
\text { maiores aumentos } \\
\text { na flexão frontal do } \\
\text { quadril. }\end{array}$ \\
\hline $\begin{array}{l}\text { HARALDSTA } \\
\text { D et al., } 2017\end{array}$ & $\begin{array}{l}\text { Descrever mudanças } \\
\text { na qualidade de vida } \\
\text { relacionada à saúde } \\
\text { (QVRS) em homens } \\
\text { mais velhos que } \\
\text { participaram de } 12 \\
\text { semanas de } \\
\text { treinamento } \\
\text { sistemático de força e } \\
\text { investigar se as } \\
\text { melhorias na força } \\
\text { muscular e na massa } \\
\text { muscular estão } \\
\text { associadas a } \\
\text { melhorias na QVRS. }\end{array}$ & $\begin{array}{l}49 \text { homens com } \\
\text { idades entre } 60 \text { e } \\
81 \text { anos } \\
\text { SF-12; }\end{array}$ & $\begin{array}{l}\text { European } \\
\text { Review Of } \\
\text { Aging And } \\
\text { Physical } \\
\text { Activity: A1 }\end{array}$ & $\begin{array}{l}\text { Programa de } \\
\text { treinamento de } \\
\text { força composto } \\
\text { por três sessões } \\
\text { por semana por } \\
\text { um período de } 12 \\
\text { semanas. }\end{array}$ & $\begin{array}{l}\text { Duas das oito } \\
\text { pontuações do } \\
\text { SF-12, a função } \\
\text { física e a saúde } \\
\text { geral e as } \\
\text { pontuações } \\
\text { resumidas dos } \\
\text { componentes } \\
\text { físicos, } \\
\text { aumentaram } \\
\text { significativament } \\
\text { e durante o } \\
\text { período de } \\
\text { intervenção. } \\
\text { Pequenas } \\
\text { correlações } \\
\text { positivas } \\
\text { significativas } \\
\text { foram } \\
\text { identificadas } \\
\text { entre melhorias } \\
\text { na força muscular } \\
\text { e melhor função } \\
\text { física e social. } \\
\text { Além disso, um } \\
\text { aumento } \\
\text { significativo na } \\
\text { massa muscular } \\
\text { total foi } \\
\text { observado } \\
\text { durante o período } \\
\text { de intervenção. }\end{array}$ & $\begin{array}{l}\text { O treinamento } \\
\text { sistemático de força } \\
\text { parece ser uma } \\
\text { intervenção } \\
\text { benéfica para } \\
\text { melhorar a QVRS, } \\
\text { força muscular e } \\
\text { massa muscular em } \\
\text { homens mais velhos }\end{array}$ \\
\hline
\end{tabular}

LEGENDAS: HD: Dinamômetro hidráulico; CD: dinamômetro dorsal; ES: Balanças Eletrônicas; PP: plataforma de pressão; FTSTS: Teste cinco vezes sentado-em-pé; SF-12: Pesquisa de Saúde de Forma Curta com 12 Itens; MMSET: Mini exame de estado mental; RCQ: razão cintura-quadril; RJP: Protocolo Rikli e Jones; 10RM: Teste Máximo de Dez Repetições; MNAC: Número máximo de repetições de curvatura do braço; SRT: teste de sentar e alcançar; MoCA: A Avaliação Cognitiva de Montreal; DXA: Análise de raios-X de dupla energia e corpo inteiro; SPPB: Bateria curta de desempenho físico; PPT: Teste de Desempenho Físico; BA: Análise de Bioimpedância; SMI: Índice de Massa Muscular Esquelética; FRT: Teste de alcance funcional; CES: Balança Eletrônica Calibrada. 
Segundo Smolarek et al. (2016), o treinamento de força além de seus efeitos positivos em idosos pode também provocar efeitos negativos no estado cognitivo caso o programa de treinamento não seja construído de uma maneira eficaz, pois os efeitos negativos do treinamento de força sobre o estado cognitivo: mudança de humor com quadro de depressão e irritabilidade.

Estudos recentes revelaram efeitos positivos do treinamento de força em relação ao aumento da força muscular em idosos. Os resultados dos estudos realizados por Mazini Filho et al. (2018) e Pinheiro et al. (2018) mostram que programas de treinamento de força com 12 semanas de duração o ganho de força acontece por intermédio das adaptações neurais, que é a ampliação da área de secção transversa do músculo, assim alcançando à hipertrofia.

Segundo Pinheiro et al. (2018) identificou no estudo que o treinamento de torça pode gerar ganhos de $3,6 \%$ a $4,5 \%$ na massa muscular, aumento de tamanho da fibra muscular tipo I de $16,45 \%$, e na fibra muscular tipo II de $24,51 \%$. Assim, recomenda-se que cada sessão de treinamento tenha de seis a oito exercícios, com três séries de 12 repetições, pelo menos 12 semanas para começar a aparecer efeitos como hipertrofia muscular, e consequente ganho de força muscular.

Contudo, conforme Mazini Filho et al. (2018), deve-se tomar cuidado com o excesso de treinamento, visto que o excesso não resulta em ganho acelerado de força muscular, pelo contrário, o treinamento de força em excesso provoca cansaço exagerado, irritabilidade, batimentos cardíacos irregulares e até crescimento anormal do tamanho do coração.

Nos estudos de Cavalcante et al. (2018), Haraldstad et al. (2017) foi possível perceber resultados positivos do treinamento força sobre a composição corporal de idosos. O treinamento com duração de 12 semanas, com frequência de duas a três vezes por semana, no estudo revela que realizando de 10 a 15 repetições é o suficiente para resultar em diminuição da gordura total em 0,04\% a 0,05\%, a androide (homem) $0,24 \%$ a $0,32 \%$, a ginoide (mulher) em $0,14 \%$ a $0,17 \%$, a do tronco em $0,07 \%$ a $0,09 \%$, e aumento da massa magra em $0,05 \%$.

Segundo Haraldstad et al. (2017), apesar dos benefícios do treinamento de força na composição corporal, é possível também ocorrer consequências danosas do treinamento na composição corporal, como a perda de massa muscular ao invés de ganho, pois isto ocorre quando o tempo de recuperação não é o suficiente e com isso os tecidos musculares não se restauram rápido o suficiente porque não existe o descanso adequado entre os treinos.

Os estudos de Lopez et al. (2017), Ribeiro et al. (2015), Stoever et al. (2018) verificaram efeitos positivos do treinamento de força sobre a fragilidade, flexibilidade e sarcopenia. No estudo demostrou que o treinamento de força é efetivo em vários aspectos. Contudo é preciso levar em conta também as implicações que o mesmo pode causar como o excesso de cargas (intensidade), pois devido à idade, os idosos vão ficando com as articulações mais frágeis e propensas a lesões, principalmente idosos que apresentem (osteoporose).

De acordo com a pesquisa de Lopez et al. (2017) os efeitos positivos do treinamento de força em idosos com fragilidade envolve a melhora de uma série de variáveis como a massa muscular, força muscular, 
potência muscular e a capacidade funcional, desde que sejam respeitadas e estudadas as variáveis do treinamento (individualidade biológica, especificidade, continuidade e reversibilidade).

No estudo de Lopez et al. (2017) foi observado melhoras na massa muscular de 3,35\% a 7,5\% após o protocolo de treinamento, em relação a força muscular, houve melhora de 6,6\% a 37,0\%, na potência muscular, melhora de até $8,2 \%$, e por fim na capacidade funcional, melhoras de 5,88\% a 14,5\% na marcha, melhoria de $23,7 \%$ no teste Sit-to-stand (STS), test performance e de $4,7 \%$ a $11,4 \%$ no Short Physical Performance Battery (SPPB).

A pesquisa realizada por Ribeiro et al. (2015) identificou que o treinamento de força promove melhora na flexibilidade. Na pesquisa foi constatado melhora na flexão cervical de $2 \%$, extensão cervical de $20 \%$, flexão frontal do quadril de $3 \%$, flexão do quadril direito de $14 \%$, flexão do quadril de $25 \%$. É evidente como o treinamento de força é um componente importante na flexibilidade de idosos, e o alongamento é normalmente utilizado como uma forma de relaxamento em uma sessão de treinamento, além disso estudos comprovam que a falta de mobilidade provoca lesões musculares, principalmente nesta população que tem grande predominância de articulações menos fortes por conta da idade, pois o músculo encurtado sobrecarrega as articulações. Porém alguns estudos defendem a ideia de que o excesso da mobilidade provoca enfraquecimento articular.

Um estudo realizado por Stoever et al. (2018), verificaram efeitos positivos do treinamento de força em idosos, onde o mesmo foi fator para melhora do quadro de sarcopenia em idosos. Houve melhora de $9 \%$ na força de preensão manual, 9,3\% na função física, 11\% no desempenho do Physical Performance Test (PPT) e de $1,4 \%$ no Functional Reach Test (FRT), todavia, o autor lembra que treinamento de força pode ser prejudicial caso o protocolo de treinamento seja montado de modo imprudente, fazendo com que piore o quadro de sarcopenia.

A explicação dos artigos selecionados neste estudo mostra que o treinamento de força é bastante eficaz na saúde de idosos, ajudando a eliminar danos causados pelo o processo de envelhecimento, possibilitando maior funcionalidade para atividades diárias como subir uma escada, levantar de uma cadeira, e também proporciona outros benefícios como melhoras na força muscular, aumento da massa muscular, redução do percentual de gordura corporal, aumento da capacidade funcional e melhora no estado cognitivo. No entanto quando o treinamento de força é feito de modo negativo não respeitando as variáveis do treinamento pode provocar complicações cognitivas e físicas.

\section{CONCLUSÕES}

Diante do estudo se conclui que o treinamento de força pode ser muito eficaz para a manutenção da saúde e uma melhor qualidade de vida, pois possibilita maior funcionalidade para atividades diárias através dos benefícios como melhoras na força, aumento da massa muscular, melhora da composição corporal, aumento da capacidade funcional reduzindo o risco de quedas e melhoras no estado cognitivo.

Cada vez mais essa população tem a necessidade de realizar um programa de treinamento de força, que vem sendo o mais recomendado por profissionais da saúde por ser um método bastante eficaz. Um 
programa combinado com treinamento de força, exercícios aeróbios e alongamentos sempre com a orientação de um profissional da área é uma excelente proposta para melhoria da saúde física e mental, em poucas semanas sua aparência física e funcionalidade estarão melhores.

Entretanto, quando o treinamento é realizado de modo não eficiente pode provocar complicações cognitivas e físicas. Sendo assim essa pesquisa contribuirá para elaboração de programas de treinamento com qualidade e mais segurança, pois a prescrição e acompanhamento deve ser de total responsabilidade do profissional da área.

São necessários novos estudos mais aprofundados que possam comprovar os benefícios do treinamento de força para a saúde de idosos, pois uma das limitações deste estudo foi a inclusão de todas as bases de dados existentes na literatura. Dessa forma não é possível garantir que todos os artigos relacionados como efeitos do treinamento de força para saúde de idosos foram analisados.

\section{REFERÊNCIAS}

ABREU, D. R. O. M.; NOVAES, E. S.; OLEIRA, R. R.; MATHIAS, T. A. F.; MARCON, S. S.. Internação e mortalidade por quedas em idosos no Brasil: análise de tendência. Ciência \& Saúde Coletiva, v.23, n.4, p.1131-1141, 2018. DOI: https://doi.org/10.1590/1413-81232018234.09962016

BARBOSA, G. F.; FARIAS, J. P. V.. O processo de envelhecimento nos dias atuais: revisão integrativa. 2019.

CYRINO, L. T.; CYRINO, E. S.; SILVA, E. C. A.; AVELAR, A.; TRINDADE, M. C. C.; SILVA, D. R. P.. Effect of 16 weeks of resistance training on strength endurance in men and women. Revista Brasileira de Medicina do Esporte, v.25, n.5, p.399-403, 2019. DOI: https://doi.org/10.1590/1517$\underline{869220192505126869}$

CARDALDA, I. M.; LÓPEZ, A.; CARRAL, J. M. C.. The effects of different types of physical exercise on physical and cognitive function in frail institutionalized older adults with mild to moderate cognitive impairment. A randomized controlled trial. Archives of Gerontology and Geriatrics, Dublin, v.83, p.223-230, 2019.

CAVALCANTE, E.; RIBEIRO, A. S.; NASCIMENTO, M. A.; SILVA, A. TOMELERI, C. M.; NABUCO, H. C. G.; PINA, F. L. C.; MAYHEW, J. L.; SILVA-GRIGOLETTO, M. E.; SILVA, D. R. P.; FLECK, S.; CYRINO, E.. Effects of Different Resistance Training Frequencies on Fat in Overweight/Obese Older Women. International Journal of Sports Medicine, Munich, v.39, n.07, p.527-534, 2018.

COLUSSI, E. L.; PICHLER, N. A.; GROCHOT, L.. Perceptions of the elderly and their relatives about aging. Revista Brasileira de Geriatria e Gerontologia, v.22, n.1, p.1-8, 2019.

CONFORTIN, S. C.; SCHNEIDER, I. J. C.; ANTES, D. L.; CEMBRANEL, F.; ONO, L. M.; MARQUES, L. P.; BORGES, L. J.; KRUG, R. R.; D'ORSI, E.. Condições de vida e saúde de idosos: resultados do estudo de coorte EpiFloripa Idoso. Epidemiologia e Serviços de Saúde, v.26, n.2, p.305317, 2017. DOI: https://doi.org/10.5123/s1679$\underline{49742017000200008}$
ESTRELA, C.. Metodologia Científica: ciência, ensino, pesquisa. 3 ed. Rio de Janeiro: Artes Médicas, 2018.

FLECK, S. J.; KRAEMER, W. J.. Fundamentos do Treinamento de Força Muscular. 4 ed. Porto Alegre: Artmed, 2017.

HARALDSTAD, K.; ROHDE, G.; STEA, T. H.; LOHNE-SEILER, H. Changes in health-related quality of life in elderly men after 12 weeks of strength training. European Review of Aging and Physical Activity, Berlin, v.14, n.1, p.1-6, 2017.

IBGE. Instituto Brasileiro de Geografia e Estatística. Pesquisa Nacional por Amostra de Domicílios Contínua. Rio de Janeiro: IBGE, 2018.

LAKATOS, E. M.; MARCONI, M. A.. Técnicas de pesquisa. 6 ed. São Paulo: Atlas, 2006.

LOPEZ, P.; PINTO, R. S.; RADAELLI, R.; RECH, A.; GRAZIOLI, R.; IZQUIERDO, M.; CADORE, E. L.. Benefits of resistance training in physically frail elderly: a systematic review. Aging Clinical and Experimental Research, Roma, v.30, n.8, p.889-899, 2017. DOI: http://doi.org/10.1007/s40520-017-0863-z

LIMA, A. P.; GIACOMAZZI, R. B.; SCORTEGAGNA, H. M.; PORTELLA, M. R.. Grupo de convivência para idosos: o papel do profissional de educação física e as motivações para adesão à prática de atividade física. Revista Brasileira de Ciências do Esporte, p.1-7, 2019. DOI: http://dx.doi.org/10.1016/j.rbce.2019.02.001

MAZINI FILHO, M.; AIDAR, F.; MATOS, D. G.; MOREIRA, O. C.; OLIVEIRA, C. E. P.; VENTURINI, G. R. O.; CURTY, V. M.; TOUGUINHA, H. M.; FERREIRA, M. E C.. Circuit strength training improves muscle strength, functional performance and anthropometric indicators in sedentary elderly women. The Journal of Sports Medicine and Physical Fitness, Milano, v.58, n.7-8, p.1029-1036, 2018.

PINTO, I. M. F.; SMANIO, P. E. P.; WILSON JUNIOR, M.. Envelhecimento: fleury medicina e saúde. Manole, 2017.

PINHEIRO, H. A.; PEREIRA, L. C.; SANTANA, F. S.; ALVES, A. T.; FACHIN-MARTINS, E.; KARNIKOWSKI, M. G. O.; MENEZES, R. 
L.. Treinamento de resistência para hipertrofia muscular em idosos. Fisioterapia Brasil, v.19, n.1, p.118-125, 2018.

RIBEIRO, A.; CARNEIRO, N. H.; NASCIMENTO, M. A.; GOBBO, L. A.; SCHOENFELDA, D.; J.; ACHOUR JÚNIOR, A.; GOBBI, S.; OLIVEIRA, A. R.; CYRINO, E. S.. Effects of different resistance training frequencies on flexibility in older women. Clinical Interventions in Aging, Wellington, v.10, p.531-538, 2015.

ROCHA, D. B.; ALVES, R. M.. Saúde pública e o processo de envelhecimento no Brasil: transformações e consequências na sociedade. Transformações e consequências na sociedade. 2019.

SANTOS, J. C. A.; RESENDE NETO, A. G.; NOGUEIRA, A.; FEITOSA NETA. M. L.. The effects of functional and traditional strength training on different strength parameters of elderly women: a randomized and controlled trial. The Journal of Sports Medicine and Physical Fitness, Milano, v.59, n.3, p.1-27, 2019.
SMOLAREK, A.; FERREIRA, L. H. B.; MASCARENHAS, L. P. G.; MCANULTY, S. R.; VERELA, K. D.; DANGUI, M. C.; BARROS, M. P.; UTTER, A. C.; SOUZA JUNIOR, T. P.. The effects of strength training on cognitive performance in elderly women. Clinical Interventions in Aging, Wellington, v.11, p.749-754, 2016.

STOEVER, K.; HEBER, A.; EICHBERG, S.; BRIXIUS, K.. Influences of Resistance Training on Physical Function in Older, Obese Men and Women with Sarcopenia. Journal of Geriatric Physical Therapy, Boston, v.41, n.1, p.20-27, 2018.

SILVA, M. J.; SILVA, G. R.. Envelhecimento e a prática de exercício físico para melhor qualidade de vida: uma revisão literária. Revista Gestão Universitária, v.10, n.5, p.126-132, 2019.

WHO. World Health Organization. Global Report on Falls Prevention in Older Age. Genebra: WHO, 2008.

A CBPC - Companhia Brasileira de Produção Científica (CNPJ: 11.221.422/0001-03) detém os direitos materiais desta publicação. Os direitos referem-se à publicação do trabalho em qualquer parte do mundo, incluindo os direitos às renovações, expansões e disseminações da contribuição, bem como outros direitos subsidiários. Todos os trabalhos publicados eletronicamente poderão posteriormente ser publicados em coletâneas impressas sob coordenação da Cognitionis Publishing, da Companhia Brasileira de Produção Científica e seus parceiros autorizados. Os (as) autores (as) preservam os direitos autorais, mas não têm permissão para a publicação da contribuição em outro meio, impresso ou digital, em português ou em tradução. 